\title{
Why Our Microbiome is Important to Our Physiology and Diseases
}

\section{Regina Au}

Principal, New Product Planning/Strategic Commercial Consultant BioMarketing Insight, Boston, Massachusetts, USA

\section{Abstract}

The human microbiome, or microbiota ecosystem, has always coexisted in harmony with our immune system but had gone unacknowledged because we didn't understand its importance until recently. Scientists have found that the human microbiome plays a critical role in human survival. The microbiome can influence our physiology, our immune system and our risk of developing diseases. Diet plays a role in establishing our initial microbiome ecology in infancy and diet continues to influence our microbiome ecology throughout our life. This article will review the findings from the Human Microbiome Project (HMP) and from various studies demonstrate how our microbiome influences our physiology and correlates to different diseases.

\section{Publication History:}

Received: December 16, 2016

Accepted: March 06, 2017

Published: March 08, 2017

\section{Keywords:}

Microbiome, Immune system, Ecology, Dose-effect relation

\section{Introduction}

In 2001, Nobel Laureate, Joshua Lederberg, an American molecular biologist, coined the term microbiome as the ecological community of commensal, symbiotic, and pathogenic microorganisms that inhabit and share the space in our body, but the microbiome was ignored as a determinant of health and disease [1]. He argued that microorganisms inhabiting the human body should be included as part of the human genome, because of their influence on human physiology, our wellbeing and our diseases [2].

This review will cover: 1) Definition of microbiome; 2) Findings from the Human Microbiome Project (HMP) and others; 3) How the microbiome influences our physiology; 4) How diet influences our microbiome and interacts with our gut; 5) Microbiome and its correlation to different diseases.

\section{Definition of microbiome}

The microbiome is defined as a community of microorganisms (bacteria, fungi and viruses) and their genetic material that coexists with a host. The number of microbes in our body out numbers human cells by 10 to 1 . Our body contains about 10 trillion human cells and we carry about 100 trillion bacterial cells [3]. However, the entire microbiome weighs only about 200 grams $(7.1 \mathrm{oz})$, with some estimates as high as 3 pounds (approximately $48 \mathrm{oz}$ ) [4]. The human microbiome (formerly known as human microbiota) resides on the surface and in deep layers of the skin, nasal and oral cavity, urogenital and gastrointestinal (GI) tract. The GI tract is the most densely colonized area, with the colon alone harboring over $10^{10}-10^{12}$ colony-forming units per gram of feces, or $70 \%$ of all microbes in the human body [2].

\section{Findings from the Human Microbiome Project and others}

In 2007, the National Institutes of Health funded the Human Microbiome Project and engaged a consortium of researchers to map or sequence the normal microbial make-up of healthy humans and in 2012 published their findings [5]:

1) HMP researchers discovered more than 10,000 microbial species that occupy the human ecosystem, accounting for approximately $81 \%$ to $99 \%$ of all microorganismal genera in healthy adults. Now that the normal microbial variations for a healthy Western population have been established, further studies will

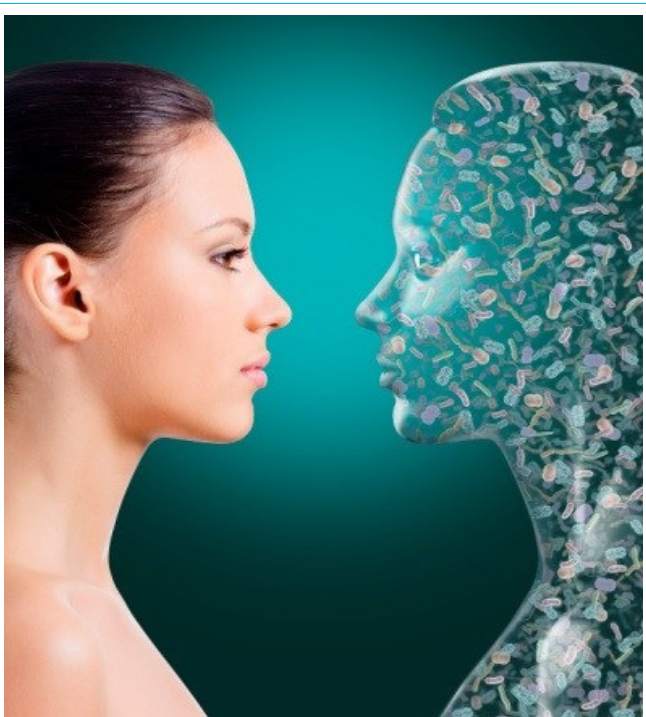

"Our Self-Portrait: the Human Microbiome," our microbiome resides on the surface and in deep layers of the skin, nasal and oral cavity, urogenital and gastrointestinal (GI) tract. Image courtesy of Broad Institute and Steven H. Lee (graphiko.com).

be conducted to determine how changes in the microbiome correlate with physiology and disease.

2) HMP researchers found that there are more genes from our microbes responsible for human survival than humans genes. The human genome carries about 22,000 protein-coding genes, while the human microbiome contributes about 8 million unique proteincoding genes, or 360 times more bacterial genes than human genes.

3) HMP researchers also found that genes from bacteria in the GI tract

"Corresponding Author: Dr. Regina Au, Principal, New Product Planning/ Strategic Commercial Consultant BioMarketing Insight, Boston, Massachusetts, USA, E-mail: regina@biomarketinginsight.com

Citation: Au R (2017) Why Our Microbiome is Important to Our Physiology and Diseases. Int $\mathrm{J}$ Clin Pharmacol Pharmacother 2: 125. doi: https://doi. org/10.15344/2017/2456-3501/125

Copyright: (C) $2017 \mathrm{Au}$. This is an open-access article distributed under the terms of the Creative Commons Attribution License, which permits unrestricted use distribution, and reproduction in any medium, provided the original author and source are credited. 
allow humans to digest foods by breaking down proteins, lipids and carbohydrates in order for human to absorb these nutrients. Since humans don't have all the necessary enzymes to digest food, these bacteria are critical for human survival. Microbes in the gut also produce beneficial compounds, including vitamins and antiinflammatories, which our genome can't produce [5], according to Lita Proctor, Ph.D., NHGRI's HMP program manager.

4) HMP Researchers discovered that the microbial metabolic activities matters more than the microbial species that perform these activities. In a healthy gut, a population of bacteria is needed to help digest fats, but the bacterial species performing this job may not always be the same. The distribution of microbial species may change over time, as humans go from a healthy state to a disease state and then back to a healthy state-- but the function of the microbes will always remain the same.

Others have found that no two individuals share the same make-up of microbes and their genes, not even identical twins, an acknowledgement of the complexity and interconnectedness of this inner ecosystem of microbiome discussed by Joshua Lederberg [6].

With high-throughput screening and sequencing of the $16 \mathrm{~S}$ ribosomal ribonucleic acid (RNA) gene content of microbes, scientists have been able to characterize the microbiome at the following anatomical sites: hair, nostril, skin, vagina, oral cavity, esophagus, and stomach for Helicobacter pylori (H. pylori) and colon. Scientists found that there are vast compositional differences in bacterial phyla (Actinobacteria, Firmicutes, Proteobacteria, Bacteroidetes, Cynobacteria, and Fusobacteria) at the various anatomical sites of an individual, as well as differences between individuals at the same anatomical sites $[7,8]$. An interesting finding revealed that there was a difference in composition in bacterial phyla for individuals that were H. pylori negative vs. H. pylori positive. H. pylori infection has been known to cause gastritis, ulcers and gastrointestinal cancer. More research is needed to determine the bacterial composition at the time of infection and what causes this shift for $\mathrm{H}$. pylori to bloom into an infection.

\section{How our microbiome influences our physiology}

The vast majority of the human microbiome is comprised of humanassociated bacterial species in terms of microbial deoxyribonucleic acid (DNA) content and cell count [2]. Early on in life the human microbiome is established and influenced by variables such as our mother's weight and diet, the way we are delivered (vaginal vs. Caesarean section (C-section)), and the foods we eat. These are among the factors that determine the composition of bacterial diversity, which in turn affects our immune system, metabolism, etc. There is a symbiotic relationship that develops between the colonizing bacteria and our innate and adaptive immune defenses, collectively comprised of the intestinal mucosal barrier to pathogens and noxious antigens.

Deanna Gibson, PhD from British Columbia, Canada and her colleagues discussed how our microbiome plays an important role in our physiology. Our microbiome is located at the interface between our internal (intestinal epithelial cells - IECs) and external (dietary antigens) environment in our gut, forming a tripartite relationship that keeps our body healthy.

The intestinal mucosa lining is a highly selective permeable monolayer, consisting of IECs and adjacent tight junctions, and acts as the only barrier separating the microbe-rich lumen side from the sterile submucosal area [9]. Any damage to this layer or loss of integrity to the tight junctions when a disease state occurs, allows for increased passage of microorganisms and their immune-stimulating molecules to the submucosa, where they can enter into circulation, induce pro-inflammatory signaling and recruit leukocytes.

Our microbiome plays several important biological roles, including the following[9]:

1) Aiding in digestion and the absorption of nutrients from partially digested food

2) Producing short-chain fatty acids (SCFA) such as such as butyrate - a primary energy source for IECs, regulates homeostasis in the gut and inhibits intestinal cell proliferation which can reduce colitis symptoms.

3) Stimulating immune responses by releasing ligands

4) Protecting against enteropathogens by producing antimicrobial peptides (AMPs)

5) Regulating the goblet cells to secret mucus droplets that replenish the mucus layer covering the epithelium

6) Acting (commensal bacteria) as a protective barrier against pathobionts (any disease-causing microorganism) by competing for food and space.

7) Regulating host fat storage [8].

The intestinal microbiome plays a crucial role in the GI tract development, systemic immunity and colonic homeostasis by interacting with the intestinal epithelial cells via the innate immune receptors. The gut associated lymphoid tissue (GALT) relays signals from the mucosal surface to the rest of the body through various immune cells and immune receptors, including innate toll- like receptors (TLRs) and NOD-like receptors (NLRs) [9]. The gut microbiome can modulate the intestinal immune cells, such as the $\mathrm{T}$ regulatory cells' function and responsiveness to bacterial products. This regulatory mechanisms is required to keep the mucosal and systemic immunity in check, allowing the mucosal surfaces to tolerate harmless bacteria, yet respond to invading pathogens.

\section{How diet influences our microbiome and intestinal mucosa}

Diets indigenous to geographical location or cultural influences can alter the intestinal microbiome both ecologically and functionally, resulting in physiological reactions to the host.

Dietary antigens can interact with both the microbiome and the intestinal mucosa, initiating biological reactions in the host. Dietary antigens are absorbed as metabolites through the intestine and into the circulating fluids like blood and lymph and the chemical composition of the diet can define the gut microbial ecology. While dietary factors can directly affect the functionality of intestinal epithelial cells and the underlying immune cells, dietary antigens can also alter the intestinal ecosystem by enabling certain microbial populations to proliferate and dampening the dominance of others [9].

Studies have found that our diet can alter our intestinal ecology, particularly in infants, and these changes are associated with clinical consequences. For example, humans who consume a lot of red meat tend to have a predominantly Bacteroides-rich gut ecosystem, while vegetarians tend to have predominantly Prevotella species [9]. European children tend to have predominately Enterobacteriaceae 
species and deficient in Bacteroides, compared to rural African children whose diet is rich in fiber. High fat diets promote dysbiosis, an unfavorable alteration of the microbiome resulting in an imbalance between protective and harmful bacteria. However, studies have found that it's the type of fat consumed that is important, rather than the total calories from fat. For example, omega- 6 polyunsaturated fatty acids (PUFAs) cause more pathobionts, but isocaloric diets supplemented with omega-3 PUFA can reverse such microbial alterations in mice.

The consequences of dysbiosis, can be detrimental when pathobionts become prominent in the microbial communities, leading to an increased expression of immune-mediated and allergic disease states. Diet-induced dysbiosis is a contributing factor in the development of gastrointestinal diseases like inflammatory bowel disease, irritable bowel syndrome and colorectal cancer, as well as systemic diseases like obesity, diabetes, atherosclerosis and nonalcoholic fatty liver disease [9].

Erika Isolauri, $\mathrm{PhD}$ and colleagues at the University of Turku, Nutrition, Allergy, Mucosal Immunology and Intestinal Microbiota (NAMI) department in Finland looked at whether reconfiguring the microbiome at an early age could have a functional impact on the risk of obesity. Studies have found that a high-fat/energy (sugar) diet alters the gut microbiome composition which promotes excessive energy harvesting and storage that relates to obesity [2]. Microbial imbalance can also lead to increase gut permeability, metabolic endotoxemia, inflammation and insulin resistance.

\section{Microbiome and Its Correlation to Different Diseases}

\section{Microbiome in the GI tract and the production of serotonin}

GI inflammation is activated by immune cells from both the innate and adaptive immune system. In addition, inflammation is associated with alterations in the number of enterochromaffin (EC) cells that reside in the GI mucosa [10]. Ninety percent of the body's serotonin production comes from these EC cells in the GI tract. Serotonin is important for maintaining GI motility and restoring hemostasis after inflammation. Changes in the number of EC cells and serotonin levels are associated with a number of GI disorders, such as inflammatory bowel disease, colitis, enteric infection-induced inflammation in the gut, and inflammatory bowel syndrome.

Elaine Hsiao, $\mathrm{PhD}$, from California Institute of Technology and her team demonstrated that gut microbes do regulate serotonin biosynthesis. In their study they found that when mice were germfree, their EC cells produced $60 \%$ less serotonin than those mice with gut bacteria [11]. When bacteria was introduced to the germfree mice, production was increased. Their findings suggest that EC cells depend on microbes for serotonin production. They also found that about 20 species of spore-forming bacteria were responsible for elevating serotonin levels when added to germ-free mice.

Highlights from the study by Hsaio and her team [12]

- Gut microbes regulate levels of 5-hydroxytryptamine (5-HT) in the colon and blood

- Spore-forming bacteria modulate metabolites that promote colon 5-HT biosynthesis

- Microbiota-dependent changes in 5-HT impact GI motility and hemostasis

- Altering the microbiota could improve 5-HT-related disease symptoms

\section{Production of Serotonin and the Central Nervous System (CNS)}

In the brain, serotonin acts as a neurotransmitter to relay messages from one area of the brain to the other. While the brain produces serotonin, $90 \%$ of serotonin is produced in the GI tract for gut sensory and function and the rest is circulated as peripheral serotonin to areas such as the cardiovascular and endocrine systems and muscles. Scientists believe that low serotonin levels may lead to CNS disorders such as depression, obsessive-compulsive disorder, anxiety, panic, and even excess anger resulting from either a lack of receptor sites able to receive the serotonin; inability of serotonin to reach the receptor sites; or a shortage in tryptophan to make serotonin [13 ]. It is also believed that serotonin is important for the regeneration of brain cells throughout our lifetime. One theory for the etiology of depression is the suppression of new brain cells and that stress is the most important precipitator of depression [13].

The term "gut-brain axis" or "second brain" was coined when the link, or communication, between the nervous systems and the digestive system was recognized. Therefore, what affects the brain, affects the gut and visa versa. Hsiao et al showed that microbes are needed for EC cells to produce normal amounts of serotonin and a number of studies have suggested that these gut microbiome can have an impact on the brain and behaviors such as anxiety and depression $[14,15]$.

In the experimental autoimmune encephalomyelitis (EAE), an animal model of human multiple sclerosis study, it was found that when certain bacteria in the gut are altered this can lead to proinflammatory conditions in distal effector immune sites, and may lead to autoimmunity disease, in this case multiple sclerosis (MS). Whereas certain commensal bacteria in the right balance can be protective against inflammation in the CNS [16].

Microbiome and the impact on rheumatoid arthritis (RA)

The etiology of RA is an intricate mechanism. Earlier studies showed that gut and joint inflammation are closely related pathogenetically when B27 transgenic rats were raised in a germ-free environment do not develop inflammatory intestinal or peripheral joint disease, suggesting that the commensal gut flora play an important role in the development of arthritis [17]. Other studies showed that conventional mice vs. germ-free mice do not develop arthritis because they may have already developed a tolerance to arthritogenic epitopes, or the bacterial flora may induce cell-mediated immunity to ubiquitous bacterial peptidoglycans providing a suppressive effect on the development of the disease $[18,19]$.

Researchers have found that Toll-Like Receptors (TLR), ligands of microbial origin, bacterial DNA and peptidoglycans, as well as Epstein-Barr virus, cytomegalovirus, and parvovirus, have repeatedly been found in the joints of patients with RA, suggesting that bacterial or viral infections may be an initiating factor for RA. In addition, in the rheumatoid synovium, the presence of endogenous TLR ligands such as fibronectin fragments and heat shock proteins has been shown to mainly activate TLR4 [21]. It is theorized that microbial and hostderived TLRs may contribute to the initiation and chronic progression of RA [20].

Abdollahi-Roodsaz [20] and colleagues investigated the involvement of TLR2, TLR4 and TLR9 in the initiation and progression of RA with Interleukin -1 receptor antagonist-knockout (IL1rn-/-) mice that 
spontaneously develops an autoimmune $\mathrm{T}$ cell-mediated arthritis due to excessive IL-1 signaling. Germ-free mice did not develop arthritis [20]. Their study showed that TLR2 down-regulates the progression of arthritis through the dampening of Regulatory $\mathrm{T}$ cells (Tregs) and regulation of interferon gamma (IFN- $\gamma$ )-producing $\mathrm{T}$ helper 1 (Th1) cells. TLR4, in contrast, contributes to more severe disease by modulating the over-activation of Th17 cell and Interleukin (IL)-17 production. TLR9 was not involved with the expression of autoimmune arthritis. It was confirmed that endogenous TLR4 ligands were present in RA synovial fluid which drives the spontaneous production of cytokines in synovial tissue.

In the $\mathrm{K} / \mathrm{BxN}$ (expressing the KRN T cell receptor transgene and the major histocompatibility complex (MHC) class II molecule $\mathrm{A}(\mathrm{g} 7)$ ) mouse model prone to arthritis, autoimmunity was attenuated in a germ-free $\mathrm{K} / \mathrm{BxN}$ mouse, implicating the importance of gut microbiome [22]. This was accompanied by reductions in serum autoantibody titers, splenic autoantibody-secreting cells, germinal centers and the splenic T helper 17 (Th17) cell population.

In both models, it is the over-activation of Th17 cells and the dampening of Treg function (via dendritic cells and TLR signaling) that drive systemic autoimmune disease [3]. This proinflammatory Th17-driven state that ultimately promote distal autoimmunity, is not unique to RA. It was shown in the experimental autoimmune encephalomyelitis (EAE) model for MS mentioned earlier, that MS is abrogated in germ-free conditions, and also with antibiotic or probiotic treatment. Tolerogenic Tregs are responsible for these protective responses [3]. EAE susceptibility and severity are enhanced by the administration of bacteria such as Porphyromonas gingivalis and Segmented Filamentous Bacteria.

\section{Microbiome in obesity and other metabolic diseases}

In previous studies, it's been shown that a change in diet can alter the gut microbiome. In some animal studies, it was shown that obese mice or mice fed a high-fat diet had a decrease in Bacteroidetes and increase in Firmicutes [23]. In a single day, a change from a low- fat to a highfat, high-sugar diet exhibited a change in microbiome that resulted in metabolic change, and an increase in adiposity and the same trait was evident with a microbiome transplant to other mice. The microbiome controls many different facets of the host metabolism, including Tlr5 activation (e.g., through bacterial flagellin) on epithelial or myeloid cells, that ultimately regulates appetite, weight gain, and insulin sensitivity and also regulates Fiaf release from intestinal epithelial cells, which acts as an inhibitor of $\mathrm{Lpl}$ and thereby regulates peripheral fat storage, both of unknown mechanism [23].

It is hypothesized that our microbiota also affects Ampk, a key enzyme that controls cellular energy like a fuel gauge. It signals to the liver and skeletal muscle and affects the skeletal muscle and liver fatty acid oxidation pathways [23]. The exact mechanism is not clear. How the microbiome affects the host metabolism is very complex, as demonstrated here, but scientists are making strides in uncovering the physiology of the interactions between gut microbiome and other metabolic diseases.

\section{Microbiome and its relationship to colorectal cancer}

Ashlee Earl, PhD from the Broad Institute in Massachusetts and colleagues examined the role of the microbiome in patients with colorectal cancer (Figure 1). They characterized the composition of

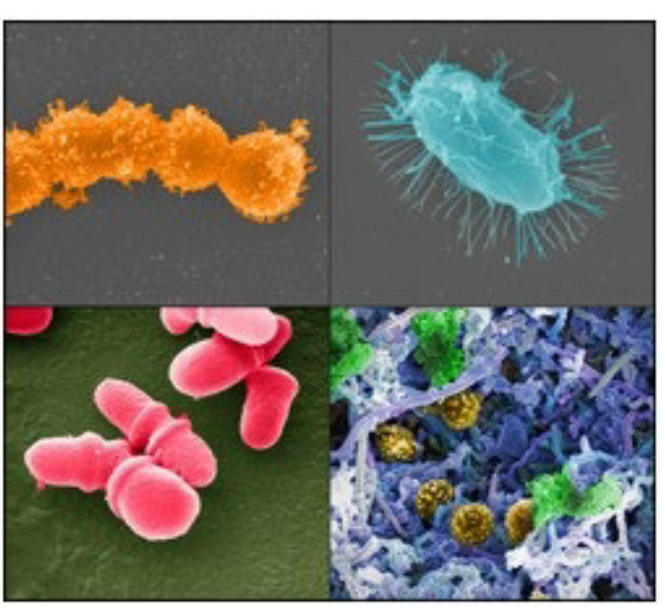

Figure 1: Mixed microbial species $(\mathrm{a}, \mathrm{b}, \mathrm{c})$ in a normal state and an unhealthy biofilm with a mixture of rods and cocci (d) causing gingival plaque. From A. Earl (Broad Institute/MIT, 2012).

the microbiota in colorectal carcinoma using whole genome sequences from nine tumor/normal pairs [24]. They found four species within the Fusobacterium genus; Fusobacterium nucleatum, Fusobacterium necrophorum, Fusobacterium mortiferum, and Fusobacterium perfoetens with F. Nucleatum being the most dominant phylotype identified within cancers. The Fusobacterium species can elicit host proinflammatory response and invade epithelial cells. The absence of Bacteroidetes and Firmicutes phyla, most prominently Clostridia, in tumors was also noted. This suggested that the dominance of Fusobacterium and the dampening of Bacteroidetes and Firmicutes sets the stage for tumorgenesis by eliciting a proinflammatory response in a subset of patients and may explains why some patients get colorectal cancer and others don't. Another hypothesis is that Fusobacterium accumulates in the tumor environment, but is not involved with the development of tumors. More research is needed to confirm either hypothesis.

Others have noted that Fusobacterium species may be associated with inflammatory bowel diseases (IBD), including both ulcerative colitis and Crohn's disease[25-27] and IBD is one of the three highest risk factors for developing colorectal cancer [24]. Likewise, more research is needed to verify a causal relationship.

\section{Conclusion}

We've known that the community of microorganisms known as the microbiome (formerly known as flora) normally resides on our skin, and it causes us no problems unless we get a cut or an open sore. When Methicillin Resistant Staph Aureus (MRSA) was a problem in hospitals, healthcare workers were found to harbor MRSA in their noses and they weren't affected, yet hospitalized patients were affected. These scenarios reinforce the commensal, symbiotic and pathogenic microorganisms that share space in and on the bodies of healthy humans. But skin microbiome, or surface microbiome, affects the host differently from gut microbiome, which affects the systemic portion of the body.

Some say that our gut microbiome is a "newly discovered organ", since its existence was not acknowledged until now. The gut microbiome is considered the "second immune system," since our exposure to the environment is through our gut with the foods we eat, the drugs we take, the environment itself (where we live and exposure 
to toxins), and the amount of stress, sleep and exercise that we receive, all of which affect our immune system and metabolic pathways. Others have considered the gut microbiome the "second brain," since there is a communication link between the nervous system and the digestive system coined the "gut-brain axis."

Scientists have discovered that our microbiome is vital for survival with respect to our GI development, digestion of foods and intake of nutrients, regulating our immune system (innate and adaptive responses), acting as a protective barrier against pathobionts, gut hemostasis and regulating host fat storage. Gut microbes also produce beneficial compounds, such as vitamins and anti-inflammatories, properties that our genome cannot produce, and they affect how well our immune system works. One of the factors that can alter our gut microbiome is our diet. Depending on the type of diet we consume, it can lead to either a favorable alteration, or to dysbiosis that could contribute to a number of serious diseases, including gastrointestinal disease and systemic diseases. The old saying "you are what you eat" has been validated with scientific evidence.

EC cells depend on microbes for serotonin production, and changes to the number of EC cells due to inflammation and lower levels of serotonin have been associated with IBD. Fusobacterium has been associated with IBD, one of the three highest risk factors for colorectal cancer. The study by Earl showed that in colorectal cancer, there is a dominance of Fusobacterium and the dampening of Bacteroidetes and Firmicutes, a dysbiosis of the microbiome and suggesting that it plays an important role in both IBS and colorectal cancer.

This study reinforces the HMP finding that the function of the microbes is more important than the type of species suggesting the importance in the change to the bacterial phyla composition and not the individual species. While F. Nucleatum was the dominate specie of the Fusobacterium genus for IBS and colorectal cancer, it's the dominance in conjunction with the dampening of Bacteroidetes and Firmicutes that played a role in colorectal cancer. The next important question will be, does restoring the Bacteroidetes and Firmicutes phyla as oppose to specific species within the genus return the host to a healthy state and eliminate one important risk factor for developing cancer?

For recurrent Clostridium difficile infections, there is a growing adoption to use fecal microbiota transplant from a healthy person as a treatment due to the dysbiosis of the microbiome in the infected patient. Currently, it's the treatment of last resort. On average the success rate is $91 \%-93 \%[28,29]$.

In the case of RA, the microbiome seems to play an integral role in the immune system, where TLR2 down-regulates the progression of arthritis and TLR4 initiates the over-activation of Th17 that is involved in more severe cases of RA.

The complexity of how the microbiome is involved with our metabolic system and its effects on our immune, cardiovascular and endocrine systems and muscles is daunting. Joshua Lederberg's foresight was accurate back in 2001, when he coined the term microbiome and recommended that it be included in the human genome because of its influence on human physiology.

These findings confirm the belief that treating patients in a holistic or multi-disciplinary approach, rather than a pure specialty approach will likely lead to better outcomes. For example, many believe that when treating CNS disorders such as depression and anxiety, one needs to treat the brain and gut simultaneously, since they are linked.
As noted earlier, it has been demonstrated that patients with depression typically have low serotonin levels and treating those patients with Selective Serotonin Reuptake Inhibitors (SSRIs) is believed to increase serotonin levels by diverting serotonin away from the gut and into the brain[30]. Serotonin is important for GI motility and hemostasis, particularly after inflammation. It has been shown that changes in the number of EC cells and serotonin levels are associated with GI disorders and could explain why SSRI users experience nausea, stomach upset, constipation, diarrhea, and fluctuations in appetite.

A study published in the New England Journal of Medicine examined two durations of antibiotic therapy for complicated abdominal infections at leading academic institutions [31]. After initial treatment, patients were randomized into one of two groups: the control arm received intravenous antibiotics until afebrile, with a normal white blood cell count and the ability to consume $50 \%$ of recommended caloric intake by mouth; the experimental arm received a 4-day short course of antibiotics regardless of physiologic parameters. Primary outcomes included surgical site infection, recurrent abdominal infection, and death [32]. The results of the trial showed that there was no difference in outcome between the standard treatment protocol and the short course of therapy and may encourage healthcare professionals to reconsider the standard practice of continuing antibiotic treatment until all physiological signs are normal. It can be theorized that a shorter course of therapy may alter the microbiome to a lesser degree and decrease the potential for negative consequences that may occur with a longer course of therapy.

Diet plays an important role in shaping the composition of the gut microbiome and this should be the first place to start, particularly since diet is linked to obesity which is then linked to Type 2 diabetes, cardiovascular disease and even cancer. To avoid gut dysbiosis, other non- pharmacological measures should be recommended as well such as exercise, which increases serotonin levels and works to alleviate depression. Life style, sleep, stress, exercise and environment each play equally important roles in our health. Unfortunately our Western life style is highly stressful and we have an appetite for instant gratification, which is reflected in our diet and other behaviors. For this reason, there is a new breed of physicians on the horizon who practice Functional Medicine that takes all these factors into consideration when treating a patient.

Pharma/biotech companies would do well to include microbiome hemostasis as part of the drug discovery process. Some companies have developed a microbiome platform, most notably Second Genome, a company that is translating novel disease insight from its platform and is targeting new disease pathways for therapeutic treatments. Other companies, such as Synthetic Biologic Inc., are developing drugs to be co-administered during antibiotic treatments, including one that protects the microbiome to help to alleviate diarrhea, a side effects of antibiotic usage that occurs as a result of dysbiosis to the microbiome. Recently, Axial Biotherapeutics is using microbiome to alleviate neurological disorder such as autism as demonstrated with mice reinforcing the gut-brain axis theory [33]. To date, there are more than 40 companies worldwide that are working in the microbiome field and the US has the most companies doing research in this area.

The field of gut microbiome research is in its infancy and it will continue to gain traction in the years to come. President Obama launched a national microbiome initiative with $\$ 121$ million in federal funding and the Bill and Linda Gates foundation and other contributed $\$ 400$ million for research in this area. By understanding how our microbiome interacts with our physiology and its correlations 
Citation: Au R (2017) Why Our Microbiome is Important to Our Physiology and Diseases. Int J Clin Pharmacol Pharmacother 2: 125. doi: https://oi. org/10.15344/2017/2456-3501/125

to diseases, we will understand diseases better, deliver better patients care and develop drugs that treat the etiology, rather than the symptoms.

\section{Conflict of Interests}

The author declares that there is no conflict of interest regarding the publication of this paper.

\section{Acknowledgements}

The author is thankful to the Broad Institute for their image and Ashlee Earl, Broad Institute for her contribution to this article.

\section{References}

1. Lederberg J, McCray A (2001) 'Ome sweet 'omics-A genealogical treasury of words. The Scientist 15: 8 .

2. Lifschitz C (2013) Annales Nestle Editorial. Ann Nutr Metab 63: 5-6.

3. Scher JU, Abramson SB (2011) The Microbiome, the Rheumatologist.

4. C Diff Foundation (2014) Microbiome and Microbiota Basics.

5. National Institute of Health news (2012) NIH Human Microbiome Project defines normal bacterial makeup of the body.

6. DiChristina M (2012) Your Microbiome Community Brings New Meaning to "We the People", Scientific American.

7. Cho I, Blazer M J (2012) The Human Microbiome: at the interface of health and disease. Nature Review Genetics13: 260-270.

8. Andersson AF, Lindberg M, Jakobsson H, Bäckhed F, Nyrén P. et al (2008) Comparative Analysis of Human Gut Microbiota by Barcoded Pyrosequencing. PLoS ONE 3: e2836.

9. Chan YK, Estaki M, Gibson DL (2013) Clinical Consequences of DietInduced Dysbiosis, Ann Nutr Metab 63: 28-40.

10. Li N, Ghia JE, Wang H, McClemens J, Cote F, et al. 2011 Serotonin Activates Dendritic Cell Function in the Context of Gut Inflammation. Am J Pathol 178: 662-671.

11. Genetic Engineering and Biotechnology News (GEN) 2015, Gut Microbes Aid Neurotransmitter Production, GENNews Highlights.

12. Yano JM, Yu K, Donaldson GP, Shastri GG, Ann P, et al. (2015) Indigenous Bacteria from the Gut Microbiota Regulate Host Serotonin Biosynthesis, Cell 161: 264-276.

13. Bouchez C (2005-2015) Serotonin: 9 Questions and Answers.

14. Foster JA, McVey Neufeld KA (2013) Gut-brain axis: how the microbiome influences anxiety and depression. Trends Neurosci 36: 305-312.

15. Cryan JF, Dinan TG (2012) Mind-altering microorganisms: the impact of the gut microbiota on brain and behaviour.Nat Rev Neurosci 13: 701-712.

16. Ochoa-Repáraz J, Mielcarz DW, Begum-Haque S, Kasper LH (2011) Gut, bugs, and brain: role of commensal bacteria in the control of centra nervous system disease. Ann Neurol 69: 240-247.

17. Taurog JD, Richardson JA, Croft JT, Simmons WA, Zhou M, et al. (1994) The germfree state prevents development of gut and joint inflammatory disease in HLA-B27 transgenic rats. J Exp Med 180: 2359-2364.

18. van den Broek MF, van Bruggen MC, Koopman JP, Hazenberg MP, van den Berg WB (1992) Gut flora induces and maintains resistance against streptococcal cell wall-induced arthritis in F344 rats. Clin Exp Immunol 88 313-317.

19. Kohashi O, Kuwata J, Umehara K, Uemura F, Takahashi T, et al. (1979) Susceptibility to adjuvant-induced arthritis among germfree, specificpathogen-free, and conventional rats. Infect Immun 26: 791-794.

20. Abdollahi-Roodsaz S, Joosten LA, Koenders MI, Devesa I, Roelofs MF, et al. (2008) Stimulation of TLR2 and TLR4 differentially skews the balance of T cells in a mouse model of arthritis. J Clin Invest 118: 205-216.
21. Roelofs MF, Boelens WC, Joosten LA, Abdollahi-Roodsaz S, Geurts J, et al. (2006) Identification of small heat shock protein B8 (HSP22) as a novel TLR4 ligand and potential involvement in the pathogenesis of rheumatoid arthritis. J Immunol 176: 7021-7027.

22. Wu HJ, Ivanov II, Darce J, Hattori K, Shima T, et al. (2010) Gut-residing segmented filamentous bacteria drive autoimmune arthritis via T helper 17 cells. Immunity 32: 815-827.

23. Tilg H, Kaser A (2011) Gut microbiome, obesity, and metabolic dysfunction J Clin Invest 121: 2126-2132.

24. Kostic AD, Gevers D, Pedamallu CS, Michaud M, Duke F, et al. (2012) Genomic analysis identifies association of Fusobacterium with colorectal carcinoma. Genome Res 22: 292-298.

25. Neut C, Bulois P, Desreumaux P, Membré JM, Lederman E, et al. (2002) Changes in the bacterial flora of the neoterminal ileum after ileocolonic resection for Crohn's disease. Am J Gastroenterol 97: 939-946.

26. Ohkusa T, Sato N, Ogihara T, Morita K, Ogawa M, Okayasu I (2002) Fusobacterium varium localized in the colonic mucosa of patients with ulcerative colitis stimulates species-specific antibody. J Gastroenterol Hepatol 17: 849-853.

27. Strauss J, Kaplan GG, Beck PL, Rioux K, Panaccione R, et al. (2011) Invasive potential of gut mucosa-derived Fusobacterium nucleatum positively correlates with IBD status of the host. Inflamm Bowel Dis 17: 1971-1978.

28. Brandt $L$ (2012) Fecal Transplantation for the Treatment of Clostridium difficile Infection. Gastroenterol Hepatol (N Y) 8: 191-194.

29. Koenigsknecht MJ, Young VB (2013) Faecal microbiota transplantation for the treatment of recurrent Clostridium difficile infection: current promise and future needs. Curr Opin Gastroenterol 29: 628-632.

30. Point of Return (2010) Gut Health - Our Second Brain.

31. Sawyer RG, Claridge JA, Nathens AB, Rotstein OD, Duane TM, et al (2015) Trial of Short-Course Antimicrobial Therapy for Intraabdominal Infection. N Engl J Med 372: 1996-2005.

32. Sergel R (2015) Brief Antibiotic Course Effective for Gut Infections - May mean antibiotic duration can be shortened, MedPage Today.

33. Sharon G, Sampson TR, Geschwind DH, Mazmanian SK (2016) The Central Nervous System and the Gut Microbiome. Cell 167: 915-932. 